\title{
Development of a unique mouse model for pancreatic cancer lymphatic metastasis
}

\author{
JIANG LONG ${ }^{1,2,3^{*}}$, GUOPEI LUO ${ }^{1,2,3^{*}}$, CHEN LIU $^{1,2,3^{*}}$, XIAOBO CUI $^{4}$, KEI SATOH $^{4}$, ZHIWEN XIAO $^{1,2,3}$, \\ BO ZHANG ${ }^{1,2,3}$, JIN XU ${ }^{1,2,3}$, QUANXING NI ${ }^{1,2,3}, \mathrm{MIN} \mathrm{LI}^{4}$ and XIANJUN YU ${ }^{1,2,3}$ \\ ${ }^{1}$ Department of Pancreatic and Hepatobiliary Surgery, Fudan University Shanghai Cancer Center; \\ ${ }^{2}$ Department of Oncology, Shanghai Medical College, Fudan University; ${ }^{3}$ Pancreatic Cancer Institute, \\ Fudan University, Shanghai 200032, P.R. China; ${ }^{4}$ The Vivian L. Smith Department of Neurosurgery, \\ The University of Texas Medical School at Houston, Houston, TX 77030, USA
}

Received June 18, 2012; Accepted July 20, 2012

DOI: $10.3892 /$ ijo.2012.1613

\begin{abstract}
Lymphatic metastasis of pancreatic cancer is a predictor of poor prognosis. However, the molecular mechanisms are largely unknown, thus, making the development of appropriate cell lines and experimental models critically important for future investigations. The purpose of the present study was to establish a 'pancreatic cancer cell and mouse model with high lymphatic metastasis potential' for in-depth study of the underlying mechanisms. The BxPC-3-LN subline, derived from the BxPC-3 human pancreatic cancer cell line, was established through serial passages in nude mice via footpad injections. The subline was able to develop notable lymphatic metastases in $100 \%$ of the recipient mice 8 weeks after tumor cell implantation. Compared with the parental BxPC-3 cells, BxPC-3-LN cells were more aggressive, displaying invasive ultrastructure, increased migration and invasion ability, and chemoresistance. Metastasis-related gene alteration including upregulation of MMP14, MMP24, MIF and ADRM1, and downregulation of TGFB2 and ROBO1 were also observed in BxPC-3-LN cells by cDNA microarrays. Thus, the newly selected BxPC-3-LN subline can serve as a unique model for further study of lymphatic metastasis of pancreatic cancer.
\end{abstract}

\section{Introduction}

Pancreatic cancer is a very aggressive disease with a median survival of 6 months and a dismal 5-year survival rate of 5\%

Correspondence to: Dr Min Li, The Vivian L. Smith Department of Neurosurgery, The University of Texas Medical School at Houston, 6431 Fannin Street, MSE R266, Houston, TX 77030, USA

E-mail:Min.Li@uth.tmc.edu

Dr Xianjun Yu, Department of Pancreatic and Hepatobiliary Surgery, Fudan University Shanghai Cancer Center, No. 270 Dong'An Road, Xuhui District, Shanghai 200032, P.R. China

E-mail: yuxianjun88@hotmail.com

*Contributed equally

Key words: pancreatic cancer, lymphatic metastasis, mouse model
(1-3). Surgical resection remains the only curative option for pancreatic cancer (4-6). However, only $15-20 \%$ of patients undergo potentially curative surgery, as the majority of cases are unresectable at the time of diagnosis due to local major vessel invasion or remote metastases (7). Moreover, the median survival rate for those with clean microscopic surgical margins is approximately 2 years, with a 5-year survival of $15-20 \%$ (8). The malignancy of pancreatic cancer is strongly associated with its local recurrences and systemic metastases, even after curative resection for early stage pancreatic cancer $(7,9,10)$.

As one of the most important routes for recurrence and metastases in pancreatic cancer, lymphatic metastases significantly correlate with poor prognosis $(6,11-13)$. The number of metastatic lymph nodes and the ratio of metastatic lymph nodes to total number of examined lymph nodes have both been reported to significantly correlate with survival after surgical resection of pancreatic cancer $(12,14)$. Lymphatic metastases also play important roles in systemic dissemination of cancer cells, as tumor cells in lymph nodes may provide a reservoir of cells leading to distant, lethal metastases (15-17). Moreover, the lymph node may also promote metastasis formation in distant organs by lymphatic-venous communications (18). However, the mechanisms of lymphatic metastasis in pancreatic cancer have not been fully studied for the lack of animal models that faithfully replicate the process of lymphatic metastasis in humans.

In the present study, we developed a BxPC-3-LN subline from a BxPC-3 pancreatic cancer cell line by serial in vivo selection. Subsequently, we characterized the phenotypes of the BxPC3-LN cells by comparing them to the parental cells. Furthermore, to investigate the molecular mechanism in lymphatic metastasis, a comparative analysis of differential gene expression profiles in these two cell lines was performed by cDNA microarray.

\section{Materials and methods}

Cell culture and laboratory animals. The human pancreatic cancer cell line BxPC-3 was obtained from the American Type Culture Collection (Rockville, MD). Monolayer cultures of BxPC-3 cells were maintained in RPMI-1640 medium supplemented with $10 \%$ fetal bovine serum, $1 \%$ penicillin-streptomycin 
(Invitrogen, Carlsbad, CA). Cultures were maintained at $37^{\circ} \mathrm{C}$ in a humidified atmosphere of $95 \%$ air and $5 \% \mathrm{CO}_{2}$.

Seven to eight-week old immunodeficient male mice (BALB/c $\mathrm{nu} / \mathrm{nu}$ ) were purchased from Shanghai SLAC Laboratory Animal Co., Ltd., China, and housed in laminar-flow cabinets under specific pathogen-free conditions. All animal procedures were approved by the institutional animal care committee, Fudan University, China.

Establishment of new cell subline. The BxPC-3 cell subline was established by a serial in vivo selection process performed as previously described with some modifications $(19,20)$ (Fig. 1). Each male nude mouse $(n=6)$ was injected subcutaneously in the left hind footpad with $0.1 \mathrm{ml}$ of BxPC-3 cells $\left(1 \times 10^{7} / \mathrm{ml}\right)$. The mice were sacrificed at 8 weeks after tumor implantation. The possible metastatic lymph nodes, including homolateral popliteal, inguinal, iliac, para-aortic, para-renal, and mesenteric lymph nodes, were dissected and dissociated sterilely. Metastatic lymph nodes were confirmed by hematoxylin and eosin staining. The suspension was filtered using a 200-metal mesh strainer (Xinrui Biotechnology, Shanghai, China) and centrifuged at $1000 \mathrm{rcf} /$ min for $5 \mathrm{~min}$ at $4^{\circ} \mathrm{C}$. The cell pellet was resuspended in culture medium and cultivated until stable populations of human tumor cells were established under the same conditions as detailed above. Cultured tumor cells were then serially re-inoculated into new recipient mice. After five rounds of in vivo selection, a variant of the BxPC-3 human pancreatic cancer cell line (BxPC3-LN) with high lymphatic metastasis capacity was established.

Cell morphology and ultrastructure. Cell morphology was viewed and photographed under a light microscope (Estativo Eclipse TE 2000S, Nikon, Spain). For ultrastructural observation, cells were fixed with $2.5 \%$ glutaraldehyde in $0.1 \mathrm{M}$ PBS for $2 \mathrm{~h}$ at room temperature. For observation under scanning electron microscope (SEM), the specimens were immersed in isoamyl acetate, air-dried, and sputter-coated with gold before examination under the Hitachi S-520 microscope (Hitachi Ltd., Tokyo, Japan). For transmission electron microscopy (TEM), the specimens were immersed twice in absolute propylene oxide and embedded in Quetol 812. Following staining with uranyl acetate and lead citrate, the specimens were observed with a Jeol JEM-1230 electron microscope (Jeol, Tokyo, Japan) at $80 \mathrm{kV}$.

Cell cycle analysis. Cells were harvested, fixed by resuspension in $10 \mathrm{ml}$ of $70 \%$ ethanol for $30 \mathrm{~min}$, and washed in ice-cold PBS. They were incubated, washed and resuspended in DNA staining solution containing $20 \mu \mathrm{g} / \mathrm{ml}$ propidium iodide (SigmaAldrich) and $100 \mu \mathrm{g} / \mathrm{ml}$ RNase (Invitrogen). DNA content was determined by flow cytometry (BD FACS Aria, USA).

In vitro growth rate assay. To evaluate in vitro growth kinetics, growth curves were prepared for each cell line. A total of $2 \times 10^{3}$ cells/well were plated in triplicate in a 96-well plate and grown for 1-7 days. Cells were then incubated at $37^{\circ} \mathrm{C}$ for $4 \mathrm{~h}$ with $20 \mu \mathrm{l}$ of MTS (Promega) per well, and absorbance was read at $492 \mathrm{~nm}$. The number of viable cells was counted at the indicated time-points in triplicate.

Cell adhesion assay. The cell adhesion assay was performed as previously described with a slight change (21). Twenty-four-well plates were coated with $10 \mu \mathrm{g} / \mathrm{ml}$ of rat tail type IV collagen (BD Biosciences, Bedford, MA). The wells were blocked with $1 \% \mathrm{BSA}$ for $1 \mathrm{~h}$ at $37^{\circ} \mathrm{C}$. After the BSA solution was aspirated, $1 \times 10^{6}$ cells in $0.1 \%$ BSA were added to the wells in triplicate. At 15 and $30 \mathrm{~min}$, the wells were washed to remove unattached cells, incubated at $37^{\circ} \mathrm{C}$ for $4 \mathrm{~h}$ in $100 \mu \mathrm{l}$ MTS and measured for absorbance at $492 \mathrm{~nm}$. The assay was repeated at least 3 times.

Cell migration assay. The cell migration assay was performed using a 24-well transwell chamber (Corning Costar, Corning, NY) migration assay as previously described with some change (22). Briefly, exponentially growing cells were trypsinized and washed in RPMI-1640/0.1\% BSA medium twice. Cells $\left(\sim 1 \times 10^{6}\right)$ were seeded in the upper chamber of each transwell unit, which contained a polycarbonate $8-\mu \mathrm{m}$ pore membrane pre-coated with $50 \mu \mathrm{l}$ of $10 \mu \mathrm{g} / \mathrm{ml}$ fibronectin (BD Biosciences) overnight. The cells were allowed to migrate at $37^{\circ} \mathrm{C}$ and $5 \% \mathrm{CO}_{2}$ for $24 \mathrm{~h}$. The unattached cells were rinsed off with PBS and the membranes containing attached cells were fixed in 10\% formalin for $30 \mathrm{~min}$ and washed with PBS. The cells were stained with crystal violet for $20 \mathrm{~min}$ and rinsed with water. Cells on the unmigrated side were gently wiped off with a wet cotton tip applicator and the membrane was rinsed with water. The membranes containing the migrated cells were dried and mounted onto slides with permount. The number of migrated cells per high power field (hpf) was determined by averaging 20 randomly counted hpfs. The assays were performed in triplicate and were reproducible in at least three batches of independent experiments.

Cell invasion assay. Transwell chamber inserts (Corning Costar) with filter membrane pore size of $8 \mu \mathrm{m}$ were coated with $0.5 \mathrm{mg} /$ $\mathrm{ml}$ Matrigel (BD Biosciences) overnight. The membranes were rehydrated and $1 \times 10^{5}$ cells in serum-free RPMI-1640 medium were placed onto the inner chamber of each insert unit. Media with $10 \%$ FBS was placed into the outer chamber as a chemoattractant. The cells were allowed to invade at $37^{\circ} \mathrm{C}$ and $5 \% \mathrm{CO}_{2}$ for $24 \mathrm{~h}$. Cells were fixed in $10 \%$ formalin and washed with PBS. The cells were stained with crystal violet and rinsed with water. Cells on the unmigrated side were gently wiped off with a wet cotton tip applicator and the membrane was rinsed with water. The membranes containing the invaded cells were dried, and mounted onto slides with permount. The number of migrated cells per hpf was determined by averaging 20 randomly counted hpfs. The assays were performed in triplicate and were reproducible in at least three batches of independent experiments.

Drug cytotoxicity assay. Cells were plated at a density of $10^{4}$ cells per well into 96-multiwell plates and were allowed to grow for $24 \mathrm{~h}$ before the experiment. The different concentrations (from 0 to $10^{4} \mathrm{nM}$ ) of gemcitabine resuspended in $100 \mu 1$ RPMI-1640 medium were added to the cells, which were then incubated for $24 \mathrm{~h}$ at $37^{\circ} \mathrm{C}$. After initial incubation, cells were incubated at $37^{\circ} \mathrm{C}$ for $4 \mathrm{~h}$ with $20 \mu \mathrm{l}$ of MTS (Promega), and absorbance was read at $492 \mathrm{~nm}$. Chemosensitivity was expressed as the drug concentration that inhibited cell proliferation by $50 \%$ ( $\mathrm{IC}_{50}$ values). The number of viable cells was counted at the indicated time-points in triplicate. Expression of chemoresistance genes related to gemcitabine, including ribonucleotide reductase M1 (RRM-1), ribonucleotide reductase M2 (RRM-2), human equilibrative nucleoside transporter-1 (hENT-1), and 


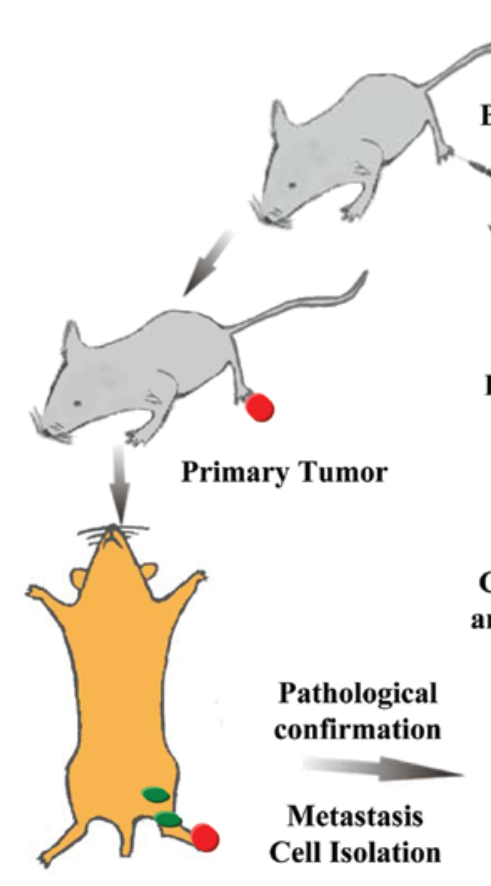

Lymph Node Metastasis
BxPC-3 Footpad Injection

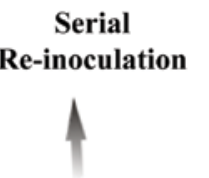

Cell Selection and Expansion

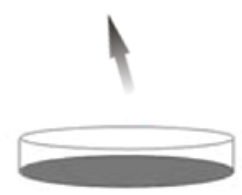

200 metal mesh strainer

Figure 1. Establishment of a highly lymphatic metastatic subline via in vivo selection. BxPC-3 cells were injected subcutaneously in the left hind footpad of male nude mice. The tumor diameter reached approximately $10-15 \mathrm{~mm}$ in 8 -weeks. Autopsy was done to remove the possible homolateral metastatic lymph nodes. Metastatic lymph nodes were confirmed by pathological confirmation. Lymph nodes were then dissected, minced, dissociated and filtered using a 200-metal mesh strainer. Cells were plated, expanded and repassaged in the animals for additional rounds.

deoxycytidine kinase (dCK), were examined by reverse-transcription polymerase chain reaction (RT-PCR) as previously described (23).

In vivo tumor growth and metastatic potential. Eight-week old mice were injected with $1 \times 10^{6} / \mathrm{ml}$ BxPC-3 and BxPC-3-LN cells into the footpad $(n=6)$. The primary tumors were allowed to form and the weight was measured every week. Tumor volume

was calculated according to the formula: (length in centimeters $\left.\mathrm{x}(\text { width })^{2}\right) / 2$. Six mice in each group were sacrificed separately at 8-week end-points to examine lymphatic involvement. The possible metastatic lymph nodes were divided into three groups: N1 (popliteal, inguinal), N2 (iliac, para-aortic), N3 (para-renal). At end-point, animals were euthanized and autopsies were performed. Organs (including lymph nodes, lungs, liver, spleen, pancreas, kidneys, ovaries, and any other tissues of abnormal appearance) were examined superficially for evidence of metastases using a dissecting microscope. Upon sacrifice, the tumors, metastatic lymph nodes, and other organs were fixed for hematoxylin and eosin staining.

cDNA microarray. Total RNA was extracted from BxPC-3 and BxPC-3-LN cells, respectively, with TRIzol (Life Technologies, USA) and dissolved in Milli-Q $\mathrm{H}_{2} \mathrm{O}$. The RNA from BxPC-3 was labeled with Cy3-dUTP and that from BxPC-3-LN with Cy5-dUTP. Fluorescent probe mixtures were denatured at $95^{\circ} \mathrm{C}$ for $5 \mathrm{~min}$, and applied to the prehybridized chip (Affymetrix, Inc., USA) under a cover glass, which was hybridized at $42^{\circ} \mathrm{C}$ for 15-20 h. After washing, the microarray was scanned on a scanner (Agilent, G2565BA) and the image was analyzed using Feature Extraction software (Agilent). The signal intensity of each spot was calibrated by subtraction from the intensity of the negative control. The assay was performed twice and only genes showing the same tendency of differential expression were eligible for the final-result analysis.

Statistical analysis. Fisher's exact test and Student's t-test were used for comparisons of enumeration data and to measure mean $\mathrm{t}$ data, respectively. The statistical analysis software package Stata 10.0 was used for the tests, and $\mathrm{P}<0.05$ was considered statistically significant.

\section{Results}

Morphology and ultrastructure. Under a light microscope, the morphology of the BxPC-3-LN cell line was similar to the parental BxPC-3 cell line observed in culture. Both of them demonstrated polygonal epithelial morphology with large nuclei
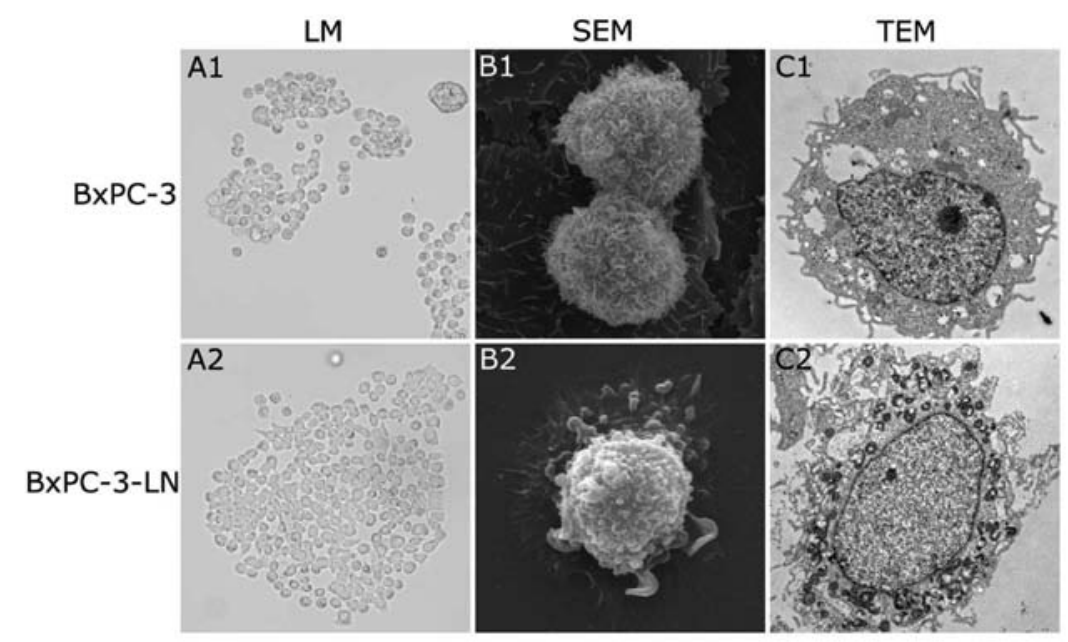

Figure 2. Morphology and ultrastructure of BxPC-3 and BxPC-3-LN cell lines. The morphology of BxPC-3-LN cells was similar to the parental BxPC-3 cells under light microscopy (A1 and A2). However, under SEM and TEM, the BxPC-3-LN cells exhibited discernible aggressive morphological alterations, such as filopodia and lamellipodia, and an increase in the number of secretory granules (B1, B2, C1 and C2). 
A

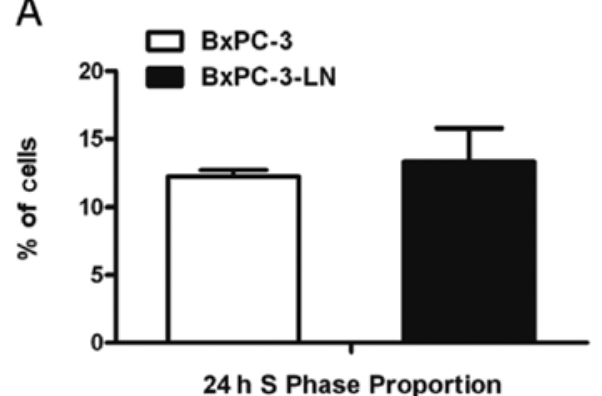

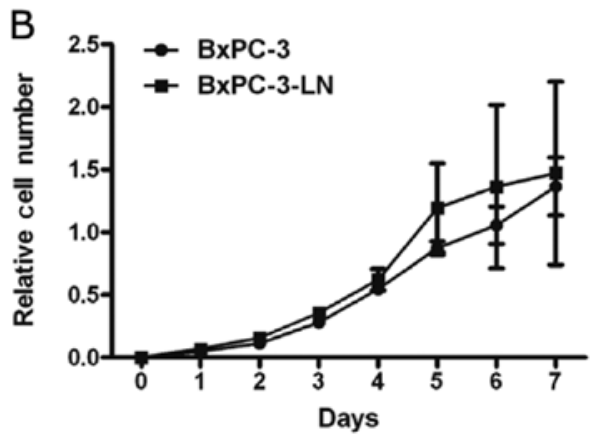

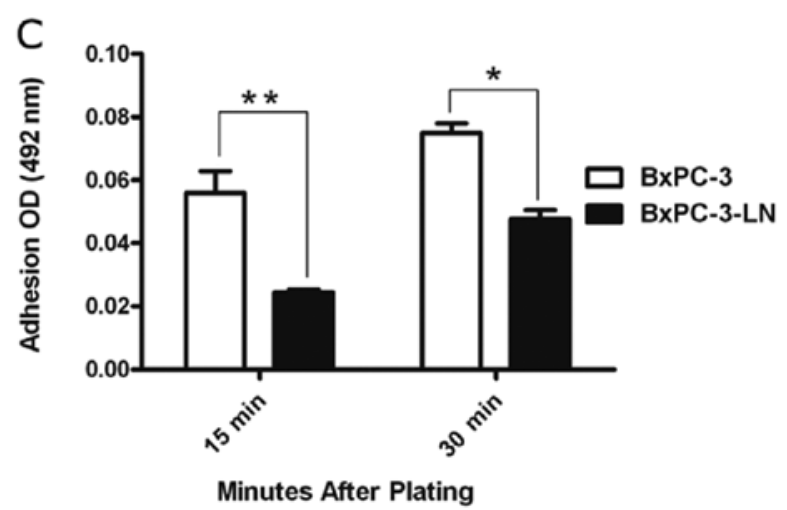

Figure 3. In vitro cell growth examined by flow cytometry, MTS assay and cell adhesion assay. The in vitro proliferation and proportion of cells in S phase was similar in BxPC-3-LN cells compared to parental cells (A and B). At the 15- and 30-min time-points, cell adhesion was significantly lower in BxPC-3-LN cells when compared to the BxPC-3 cells, ranging from 36.0 to $57.0 \%\left[(\mathrm{C})^{* * * *} \mathrm{P}<0.05\right]$.

A1

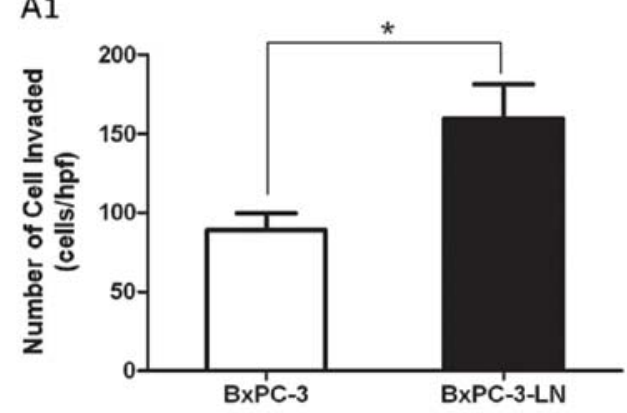

BxPC-3

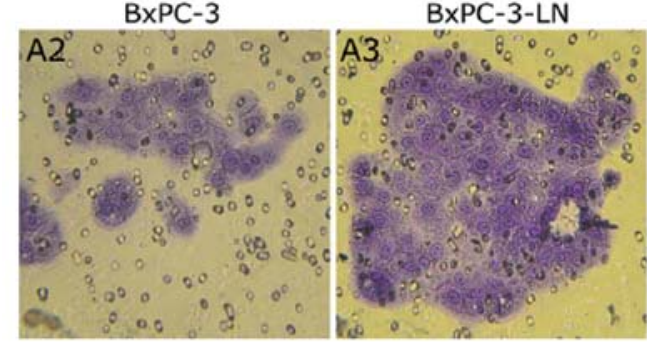

B1

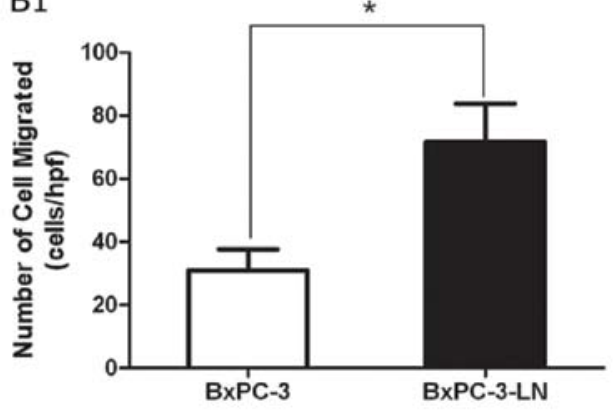

$\mathrm{BXPC}-3$

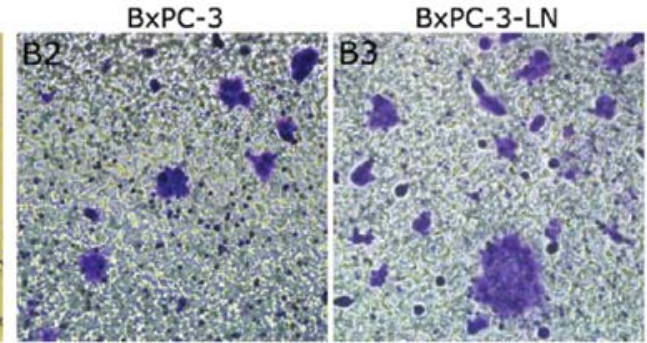

Figure 4. In vitro migration and invasion ability assay. (A and B) BxPC-3-LN cells demonstrated increased cell migration and invasion compared to the parental BxPC-3 cells. (A2, A3, B2 and B3) Representative sample of a high power field of the migrated and invaded cells for the two cell lines (x400).

and 3-6 conspicuous nucleoli (Fig. 2A1 and A2). In addition, under SEM, in comparison to the parental cells, the BxPC-3-LN cells exhibited discernible aggressive morphological alterations such as filopodia and lamellipodia, protrusions emerging from the cellular membrane (Fig. 2B1 and B2). Under TEM, compared to the parental cells, the BxPC-3-LN cells contained more secretory granules ( $\sim 50-400 \mathrm{~nm}$ in diameter) (Fig. $2 \mathrm{Cl}$ and $\mathrm{C} 2$ ).
In vitro cell growth. Cell cycle analysis by flow cytometry revealed that the percentage of BxPC-3-LN cells in S phase (13.3\%) was similar to that of the parental cells $(12.2 \%)$ (Fig. 3A). Growth curves were employed to evaluate the in vitro growth kinetics through MTS assay. BxPC-3-LN cells exhibited similar in vitro proliferation to BxPC-3 cells (Fig. 3B). 

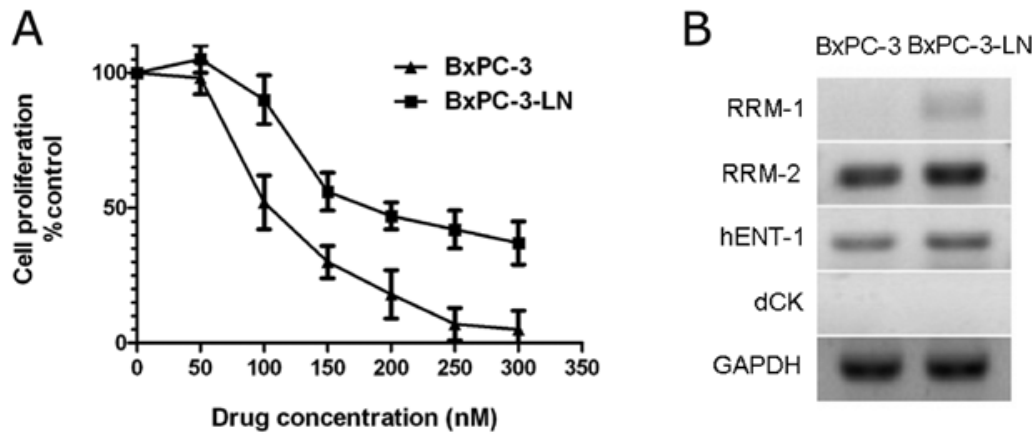

Figure 5. Drug cytotoxicity assay. (A) Cell proliferation following 24-h gemcitabine treatment was estimated by MTS assay. Quantitative values are means \pm SEM from 3 independent experiments performed in quadruplicate. (B) Expression of chemoresistance genes related to gemcitabine examined by RT-PCR. RRM-1, RRM-2 and hENT-1 expression was higher in BxPC-3-LN cells compared with that in BxPC-3 cells.

Table I. Lymphatic metastasis of each lymph node group.

\begin{tabular}{lcccccc}
\hline Cell lines & $\begin{array}{c}\mathrm{N} 1 \\
(\mathrm{M}-\mathrm{LN} / \mathrm{T}-\mathrm{LNs})(\%)\end{array}$ & $\begin{array}{c}\mathrm{N} 2 \\
(\mathrm{M}-\mathrm{LNs} / \mathrm{T}-\mathrm{LNs})(\%)\end{array}$ & $\begin{array}{c}\mathrm{N} 3 \\
(\mathrm{M}-\mathrm{LN} / \mathrm{T}-\mathrm{LNs})(\%)\end{array}$ & $\begin{array}{c}\text { All lymph nodes } \\
(\mathrm{M}-\mathrm{LNs} / \mathrm{T}-\mathrm{LNs})(\%)\end{array}$ & $\begin{array}{c}\text { Total animals } \\
(\mathrm{M} / \mathrm{T})(\%)\end{array}$ \\
\hline BxPC-3 & $1 / 12 \quad(8.3)$ & $1 / 12(8.3)$ & $0 / 12(0.0)$ & $2 / 36(5.6)$ & $1 / 6(16.7)$ \\
BxPC-3-LN & $12 / 12(100.0)$ & $10 / 14(71.4)$ & $5 / 12(41.7)$ & $27 / 38(71.1)$ & $6 / 6(100.0)$ \\
\hline
\end{tabular}

M, metastatic; T. total; LNs, lymph nodes; N1, popliteal, inguinal lymph nodes; N2, iliac, para-aortic lymph nodes; N3, para-renal lymph nodes.

Cell adhesion, migration, and invasion assay. As shown in Fig. 3C, the highly metastatic BxPC-3-LN cells had decreased relative adherence compared to the parental BxPC-3 cells. At the 15- and 30-min time-points, the decrease in relative adherence ranged from 36.0 to $57.0 \%(\mathrm{P}<0.05)$. Conversely, BxPC-3-LN cells demonstrated increased cell migration and invasion compared to the parental BxPC-3 cells (Fig. 4). In fact, when the migrated cells were counted, there was more than twice the number of cells migrated per high power field in BxPC-3-LN samples than in BxPC-3 samples (Fig. 4B1, B2 and B3).

Drug cytotoxicity assay. We examined the cellular sensitivity of BxPC-3 cells and BxPC-3-LN cells to gemcitabine in vitro using the proliferation assay. Cells were treated with increasing concentrations of gemcitabine (50-300 nM) (Fig. 5A). BxPC3-LN cells exhibited native resistance to gemcitabine and the $\mathrm{IC}_{50}$ value of BxPC-3-LN cells $(210.5 \mathrm{nM})$ was higher than that of BxPC-3 cells $(125.3 \mathrm{nM})(\mathrm{P}<0.05)$. In addition, expression of chemoresistance genes related to gemcitabine was examined by RT-PCR. RRM-1, RRM-2 and hENT-1 expression was higher in BxPC-3-LN cells compared to BxPC-3 cells (Fig. 5B).

In vivo spontaneous lymphatic metastasis via footpad injection. After five rounds of consecutive in vivo selection, the lymphatic metastases of BxPC-3-LN cells were obvious. When injected into nude mice, BxPC-3-LN cells had a $>12$-fold increase in presence of lymphatic metastases compared to the parental BxPC-3 cells (Fig. 6 and Table I). BxPC-3-LN cells also formed larger primary tumors when compared to the parental BxPC-3 cells (Fig. 6C). Total lymph node and total animal metastatic ratio of the BxPC-3-LN cells was 71.1 and $100.0 \%$, which was higher than that of the parental BxPC-3 cells (5.6 and 16.7\%, respectively) (Table I). No metastases were found in the liver, other abdominal viscera or the lungs.

Comparison of gene expression between $B x P C-3$ and $B x P C$ 3-LN cell lines. The differences in the gene expression levels between the two cell lines were assessed by measuring the ratios of their expression. Genes were identified as differentially expressed if the absolute value of the natural logarithm of the ratios was $<0.3$ or $>2.0$. Thirty-six genes were identified as being differentially expressed, including 6 with decreased expression and 30 with increased expression in BxPC-3 vs BxPC-3-LN (Fig. 7).

\section{Discussion}

Several human pancreatic cancer cell lines have been employed in studying the molecular mechanisms of pancreatic cancer lymphatic metastasis $(24,25)$. Although these cell lines show different phenotypes and genotypes that are representative of pancreatic cancer, few of them have the capability for spontaneous lymphatic metastasis in immunodeficient mice $(26,27)$. It is critical to establish appropriate cell lines and animal models to interpret the molecular mechanisms of lymphatic metastasis for pancreatic cancer.

The parental BxPC-3 cell line was first established from the body of the pancreatic adenocarcinoma of a 61-year-old female patient reported in 1986 (27). It is one of the most commonly referenced cell lines of pancreatic cancer (28). Compared to other pancreatic cancer cell lines, BxPC-3 shows moderate propensities of adhesion, migration, invasion and tumorigenicity (27). Evidence of lymphatic metastasis was rarely found to be associated with BxPC-3 (27). Therefore, it could serve as 

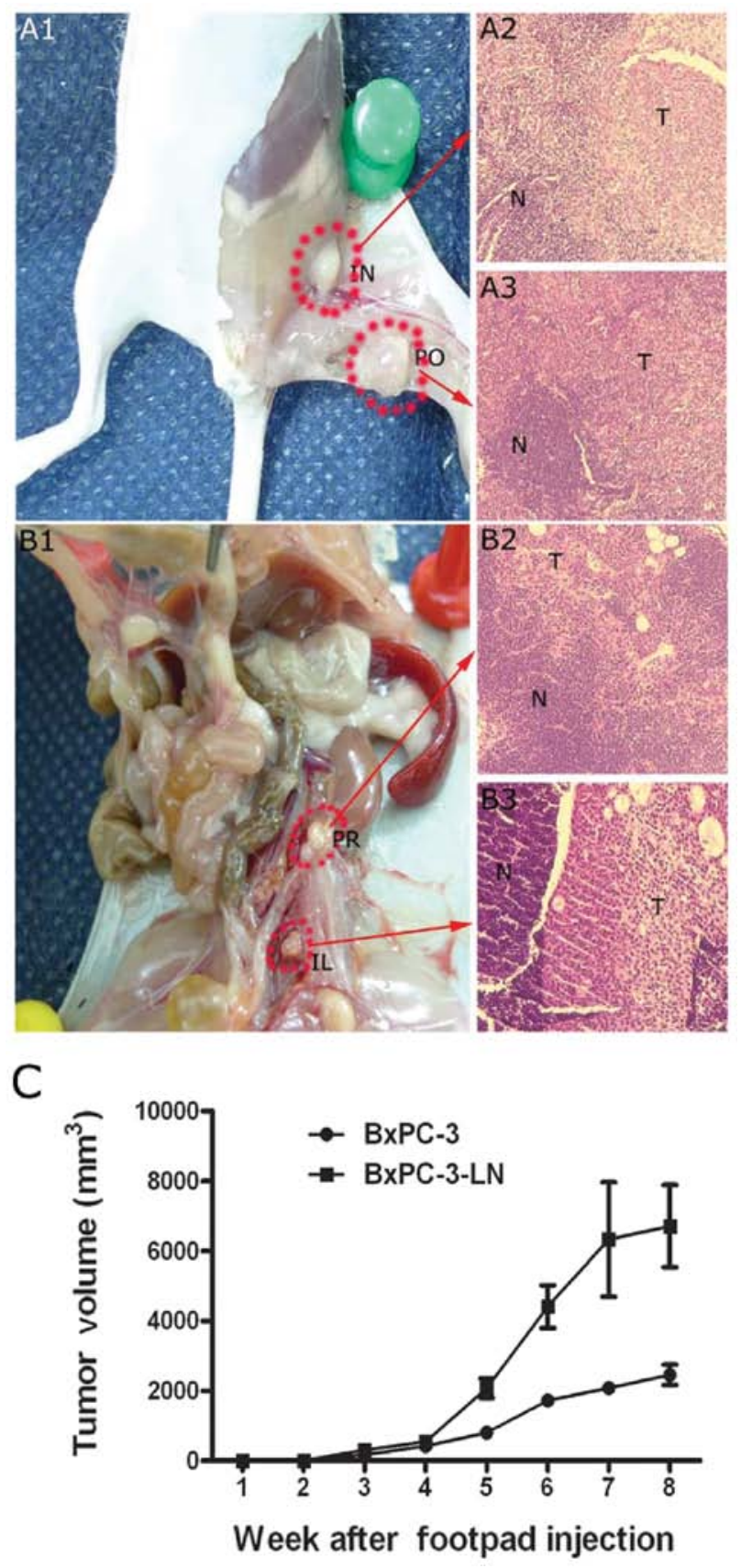

Figure 6. In vivo spontaneous lymphatic metastasis via footpad injection. (A and B) Metastatic lymph nodes (A1 and B1) confirmed by pathological examination through hematoxylin and eosin staining (A2, A3, B2 and B3) (x100). (C) A million cells were injected into the footpad of nude mice and the dimensions and volumes of the tumor were measured and calculated every week.

an ideal parental cell line from which to select highly lymphatic metastasis cell lines.

We used an in vivo selection process by injecting tumor cells into the footpad of nude mice and then examining and culturing the draining metastatic lymph nodes to establish the subline. This method was first developed by Carr et al in 1976 (19), and later confirmed as a useful and efficient method to develop lymphatic metastatic animal models $(20,29)$. The basic model is to inject tumor cells into the footpad and then to examine the draining popliteal lymph node histologically at varying times thereafter (29). We have made some modifications from the
A B

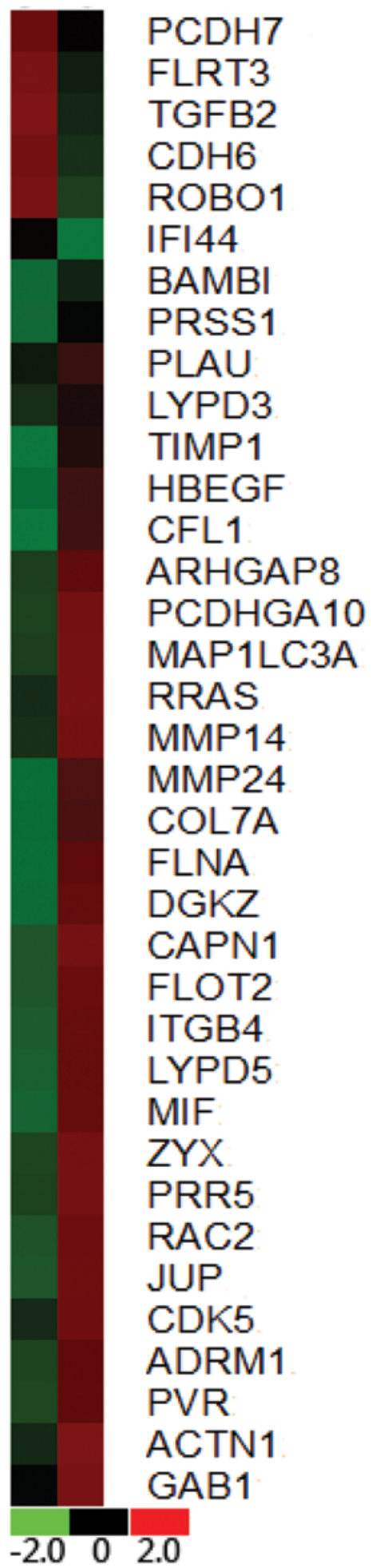

Figure 7. Comparison of gene expression by cDNA microarray. (A) BxPC-3; (B) BxPC-3-LN. Thirty-six metastasis-related genes were identified as being differentially expressed, including 6 with decreased expression and 30 with increased expression.

previous methods. First, a repetitive in vivo selection process was used to establish the high lymphatic metastasis subline. Second, lymph nodes in the lymph draining route, including popliteal, inguinal, iliac, para-aortic, or para-renal lymph nodes, were removed and pathologically confirmed. Last, but 
most importantly, we improved this technique by using a metal mesh strainer to get rid of major non-tumor cells.

After five rounds of in vivo selection, a variant of the BxPC-3 human pancreatic cancer cell line with high lymphatic metastatic potential, BxPC-3-LN, was established. Although the morphology of $\mathrm{BxPC}-3-\mathrm{LN}$ cells was similar to the parental cells by light microscopy, the ultrastructure of BxPC-3-LN cells observed under SEM and TEM showed a more aggressive change, with the presence of filopodia, lamellipodia and a greater number of secretory granules. In addition, our results demonstrated that the BxPC-3-LN cells exhibited decreased cell adhesion, and increased migration, invasion, drug resistance, and spontaneous lymphatic metastases in vivo, indicating the aggressive phenotype of the BxPC-3-LN cells.

BxPC-3-LN cells can be used as a valuable tool to study the molecular mechanisms of lymphatic metastasis for pancreatic cancer. For example, lymphatic metastatic related genes and proteins can be identified by comparing $\mathrm{BxPC}-3$ and BxPC-3-LN cells. In the present study, we compared the gene expression between BxPC-3 and BxPC-3-LN by cDNA array assay. As expected, altered expressions of numerous genes related to invasion, motility and tumorgenicity in these cells were revealed. Metastasis-related gene alteration including upregulation of MMP14, MMP24, MIF, and ADRM1, and downregulation of TGFB2 and ROBO1 were observed. Our study demonstrated that gene expression patterns undergo complex alterations during the course of lymphatic metastasis of pancreatic cancer. In addition, this model may serve as an ideal platform to study potential therapeutic approaches for lymphatic metastasis of pancreatic cancer. For example, it can be used as a model to test drugs that may potentially inhibit lymphatic metastasis and eradicate tumor cells.

In conclusion, we have developed and characterized a highly lymphatic metastatic human pancreatic cancer cell line that could serve as a useful platform for the study of pancreatic cancer lymphatic metastasis and potential therapeutics.

\section{Acknowledgements}

This study was supported in part by the National Science Foundation of China (grant nos. 81101807, 81001058, 30901435 and 30972905), the Shanghai Science and Technology Commission (10JC1418900), the MacDonald Research Fund, and the William and Ella Owens Medical Research Foundation.

\section{References}

1. Jemal A, Siegel R, Ward E, Hao Y, Xu J and Thun MJ: Cancer statistics, 2009. CA Cancer J Clin 59: 225-249, 2009.

2. Kim JW, Shin SS, Heo SH, et al: Diagnostic performance of 64-section CT using CT gastrography in preoperative T staging of gastric cancer according to 7th edition of AJCC cancer staging manual. Eur Radiol 22: 654-662, 2012.

3. Braat H, Bruno M, Kuipers EJ and Peppelenbosch MP: Pancreatic cancer: promise for personalised medicine? Cancer Lett 318: 1-8, 2012.

4. Bouvet M, Bold RJ, Lee J, et al: Adenovirus-mediated wild-type p53 tumor suppressor gene therapy induces apoptosis and suppresses growth of human pancreatic cancer. Ann Surg Oncol 5: 681-688, 1998.

5. Luo G, Long J, Zhang B, et al: Stroma and pancreatic ductal adenocarcinoma: an interaction loop. Biochim Biophys Acta 1826: $170-178,2012$.
6. Ni X, Yang J and Li M: Imaging-guided curative surgical resection of pancreatic cancer in a xenograft mouse model. Cancer Lett 324: $179-185,2012$.

7. Wray CJ, Ahmad SA, Matthews JB and Lowy AM: Surgery for pancreatic cancer: recent controversies and current practice. Gastroenterology 128: 1626-1641, 2005.

8. Geer RJ and Brennan MF: Prognostic indicators for survival after resection of pancreatic adenocarcinoma. Am J Surg 165: 63-72, 1993.

9. Warshaw AL and Fernandez-del Castillo C: Pancreatic carcinoma. N Engl J Med 326: 455-465, 1992.

10. Shi WD, Meng ZQ, Chen Z, Lin JH, Zhou ZH and Liu LM: Identification of liver metastasis-related genes in a novel human pancreatic carcinoma cell model by microarray analysis. Cancer Lett 283: 84-91, 2009.

11. Hirono S, Yamaue H, Hoshikawa Y, et al: Molecular markers associated with lymph node metastasis in pancreatic ductal adenocarcinoma by genome-wide expression profiling. Cancer Sci 101: 259-266, 2010.

12. Isaji S, Kawarada $\mathrm{Y}$ and Uemoto S: Classification of pancreatic cancer: comparison of Japanese and UICC classifications. Pancreas 28: 231-234, 2004.

13. Li Y, Kong D, Ahmad A, Bao B and Sarkar FH: Pancreatic cancer stem cells: emerging target for designing novel therapy. Cancer Lett: May 20, 2012 (Epub ahead of print).

14. Pedrazzoli S, DiCarlo V, Dionigi R, et al: Standard versus extended lymphadenectomy associated with pancreatoduodenectomy in the surgical treatment of adenocarcinoma of the head of the pancreas: a multicenter, prospective, randomized study. Lymphadenectomy Study Group. Ann Surg 228: 508-517, 1998.

15. Alitalo K, Tammela T and Petrova TV: Lymphangiogenesis in development and human disease. Nature 438: 946-953, 2005.

16. Oliver $\mathrm{G}$ and Detmar M: The rediscovery of the lymphatic system: old and new insights into the development and biological function of the lymphatic vasculature. Genes Dev 16: 773-783, 2002.

17. Zhang Y, Tang H, Cai J, et al: Ovarian cancer-associated fibroblasts contribute to epithelial ovarian carcinoma metastasis by promoting angiogenesis, lymphangiogenesis and tumor cell invasion. Cancer Lett 303: 47-55, 2011.

18. Yin T, Ji XL and Shen MS: Relationship between lymph node sinuses with blood and lymphatic metastasis of gastric cancer. World J Gastroenterol 9: 40-43, 2003.

19. Carr I, McGinty F and Norris P: The fine structure of neoplastic invasion: invasion of liver, skeletal muscle and lymphatic vessels by the Rd/3 tumour. J Pathol 118: 91-99, 1976.

20. Carr J, Carr I, Dreher B and Betts K: Lymphatic metastasis: invasion of lymphatic vessels and efflux of tumour cells in the afferent popliteal lymph as seen in the Walker rat carcinoma. J Pathol 132: 287-305, 1980.

21. Luu HH, Kang Q, Park JK, et al: An orthotopic model of human osteosarcoma growth and spontaneous pulmonary metastasis. Clin Exp Metastasis 22: 319-329, 2005.

22. Varney ML, Singh S, Li A, Mayer-Ezell R, Bond R and Singh RK: Small molecule antagonists for CXCR2 and CXCR1 inhibit human colon cancer liver metastases. Cancer Lett 300: 180-188, 2011.

23. Luo G, Jin C, Long J, et al: RNA interference of MBD1 in BxPC-3 human pancreatic cancer cells delivered by PLGA-poloxamer nanoparticles. Cancer Biol Ther 8: 594-598, 2009.

24. Ochi N, Matsuo Y, Sawai H, et al: Vascular endothelial growth factor-C secreted by pancreatic cancer cell line promotes lymphatic endothelial cell migration in an in vitro model of tumor lymphangiogenesis. Pancreas 34: 444-451, 2007.

25. Taniguchi S, Iwamura $\mathrm{T}$ and Katsuki T: Correlation between spontaneous metastatic potential and type I collagenolytic activity in a human pancreatic cancer cell line (SUIT-2) and sublines. Clin Exp Metastasis 10: 259-266, 1992.

26. Lieber M, Mazzetta J, Nelson-Rees W, Kaplan M and Todaro G: Establishment of a continuous tumor-cell line (panc-1) from a human carcinoma of the exocrine pancreas. Int J Cancer 15: 741-747, 1975.

27. Tan MH, Nowak NJ, Loor R, et al: Characterization of a new primary human pancreatic tumor line. Cancer Invest 4: 15-23, 1986.

28. Blanquicett C, Saif MW, Buchsbaum DJ, et al: Antitumor efficacy of capecitabine and celecoxib in irradiated and lead-shielded, contralateral human BxPC-3 pancreatic cancer xenografts: clinical implications of abscopal effects. Clin Cancer Res 11: 8773-8781, 2005.

29. Carr I: Experimental lymphatic metastasis. J Microsc 131: 211-220, 1983. 